\title{
New Species of the Genus Actinoplanes, Actinoplanes ferrugineus
}

\author{
NORBERTO J. PALLERONI \\ Chemical Research Department, Hoffmann-La Roche Inc., Nutley, New Jersey 07110
}

An extensive phenotypic characterization of an actinoplanete capable of producing the antimetabolite L-azetidine-2-carboxylic acid is presented. On the basis of the properties of this organism, it is concluded that it belongs to a new species, for which the name Actinoplanes ferrugineus is proposed. The type strain is X14695 (= ATCC 29868).

In the course of screening for new antibiotics, I isolated an actinoplanete which produced Lazetidine-2-carboxylic acid, a proline antimetabolite (D. Pruess and J. Scannell, personal communication). Since this compound had never before been reported as being produced by a procaryotic organism (3), it was of interest to characterize the producing strain, X-14695. Colonies of this organism have a rusty, reddishbrown color when grown on starch-casein agar. This color and the production of azetidine-carboxylic acid suggested that strain X-14695 belongs to a new species of Actinoplanes. This report provides morphological and physiological support for the recognition of a new species. The isolation, characterization, and biosynthesis of L-azetidine-2-carboxylic by this organism will be reported elsewhere.

\section{MATERIALS AND METHODS}

Organisms. Strain X-14695 of our collection was isolated in our laboratory by means of a novel method (to be described in detail elsewhere) for the isolation of actinoplanetes. The source of the culture was a red soil sample collected by T. Daniels at Dorrigo Mountain, Australia. Actinoplanes brasiliensis ATCC 25844, A. italicus ATCC 27366, and A. utahensis ATCC 14539 were purchased from the American Type Culture Collection (ATCC), Rockville, Md.

Culture methods and nutritional screening. To determine the growth chacteristics of, and color production by, this organism, it was grown in several of the culture media recommended by Shirling and Gottlieb (8) for streptomycetes. Other media used were nutrient agar (Difco Laboratories), the medium described by Hickey and Tresner (4), glucose-asparagine agar, potato-dextrose agar, and Czapek-glucose agar prepared by the method of Waksman (11). The medium routinely used for maintenance of the cultures was the starch-casein agar of Waksman (11), modified by the addition of $0.05 \% \quad \mathrm{MgSO}_{4}$ (or $0.102 \%$ $\mathrm{MgSO}_{4} \cdot 7 \mathrm{H}_{2} \mathrm{O}$ ).

Growth on a large number (140) of low-molecularweight organic compounds was determined by the method of Prosser and Palleroni (7). Individual carbon sources were added to the basal mineral agar to a final concentration of $0.1 \%$. Geraniol and naphthalene were not incorporated into the medium but instead were placed in the lids of the petri dishes; after inoculation, the plates were individually wrapped in plastic bags for incubation. A plate with no added carbon source was used as a control of background growth.

Screening for utilizable substrates (with the exception of geraniol and naphthalene) was also performed in liquid mineral medium dispensed in tubes $(4 \mathrm{ml}$ of medium in each test tube [ 20 by $150 \mathrm{~mm}$ ]); the inoculated tubes were incubated in a water bath with rotary shaking $(250 \mathrm{rpm})$ at $28^{\circ} \mathrm{C}$.

The following organic compounds were tested as single sources of carbon: D-ribose, D-xylose, D-arabinose, L-arabinose, $D$-fucose, L-fucose, L-rhamnose, $D$ glucose, D-mannose, D-galactose, D-fructose, L-sorbose, sucrose, trehalose, maltose, cellobiose, lactose, melibiose, salicin, raffinose, melezitose, starch, chitin, gluconate, 2-ketogluconate, saccharate, mucate, lactobionate, formate, acetate, propionate, butyrate, isobutyrate, valerate, caproate, heptanoate, caprylate, pelargonate, caprate, oxalate, succinate, malonate, maleate, fumarate, glutarate, adipate, pimelate, suberate, sebacate, tartronate, D-malate, L-malate, D-(-)-tartrate, L-(+)-tartrate, $m$-tartrate, beta-hydroxybutyrate, DL-lactate, glycolate, glycerate, citrate, alpha-ketoglutarate, pyruvate, aconitate, levulinate, itaconate, ethylene glycol, glycerol, propylene glycol, erythritol, 2,3-butylene glycol, D-arabitol, xylitol, dulcitol, adonitol, mannitol, sorbitol, $m$-inositol, methanol, ethanol, $n$-propanol, isopropanol, $n$-butanol, isobutanol, secbutanol, geraniol, L-mandelate, benzoate, phenylacetate, naphthalene, phenol, quinate, glycine, L-alanine, D-alanine, beta-alanine, L-threonine, L-leucine, $\mathrm{L}$-isoleucine, DL-norleucine, L-valine, L-aspartate, D-aspartate, L-glutamate, L-asparagine, L-glutamine, L-lysine, L-arginine, L-citrulline, L-ornithine, alpha-aminobutyrate, gamma-aminobutyrate, alpha-aminovalerate, delta-aminovalerate, L-homoserine, L-methionine, Lhistidine, L-proline, L-hydroxyproline, L-tyrosine, Lphenylalanine, L-tryptophan, kynurenate, anthranilate, methylamine, ethanolamine, putrescine, spermine, butylamine, sec-butylamine, tert-butylamine, alpha-amylamine, betaine, sarcosine, creatine, hippurate, pantothenate, acetamide, nicotinate, isonicotinate, and trigonelline. Sodium salts of the anionic substrates were prepared by neutralization of the free acids with sodium hydroxide. All compounds were reagent grade. 
The inoculum for the various media was a washed suspension of spores. Good sporulation was obtained on mineral agar with $0.1 \%$ L-aspartate. Lawns of the organism on the surface of this medium were flooded with sterile $0.005 \mathrm{M}$ phosphate buffer ( $\mathrm{pH} 6.8$ ). About $1 \mathrm{~h}$ later the suspension of motile spores was collected, and the spores were washed once with buffer of the same composition. The washed spores were finally suspended in buffer to a concentration of about $10^{7}$ to $10^{8} / \mathrm{ml}$. Small drops of this suspension were used to inoculate the plates or the liquid media.

Growth characteristics on the various carbon sources were determined after incubation for 14 days at $28^{\circ} \mathrm{C}$. Colors were determined according to the Color Harmony Manual (4th ed., Container Corporation of America, Chicago, Ill., 1958). The plates were examined under a microscope with low magnification $(\times 100$ to $\times 200)$ for the presence of spores in the patches.

Physiological tests. Gelatin hydrolysis was determined by the method in Skerman's Guide (9); the medium contained Difco Thermoactinomyces fermentation broth instead of nutrient broth. Casein hydrolysis was tested in this same medium supplemented with $0.2 \%$ casein. Temperature relationships were determined in Thermoactinomyces fermentation broth, and the cultures were incubated in a well-thermoregulated water bath with rotary shaking $(250 \mathrm{rpm})$.

Analysis of carotenoid pigments. A pellet of cells of strain X-14695 was extracted with methanol under a nitrogen atmosphere, and the extract was scanned with a Gilford 250 recording spectrophotometer in the visible region of the spectrum between 400 and $600 \mathrm{~nm}$.

Cell wall analyses. Cell wall analyses were performed on cell walls isolated by the method of Yamaguchi (12). Hydrolyzed cell walls were analyzed by the procedure of Becker et al. (1). Electron micrographs of platinum-shadowed spores were kindly made by Frank Jenkins of the Roche Institute of Molecular Biology. Chemotactic behavior of the spores was studied by the method of Palleroni $(5,6)$.

\section{RESULTS AND DISCUSSION}

Morphological properties. The characteristics of strain X-14695 are as follows. Growth occurred on many media currently used for actinomycetes. The colonies on many of these media were brown to dark red (mahogany) in color (see below). When observed under the microscope with low magnification, sporangia were visible in many cases (Fig. 1). These sporangia had diameters which ranged from 4 to 12 $\mu \mathrm{m}$. The formation of sporangia by strain X14695 was far less abundant and reproducible in comparison with other actinoplanetes, and the sporangia were more difficult to observe. Thus, whereas $A$. brasiliensis ATCC 25844, A. italicus ATCC 27366, and A. utahensis ATCC 14539 formed sporangia very readily in various standard media, and the numerous sporangia on the upper surface of the colonies could be easily observed with a standard dissecting microscope, the sporangia of strain X-14695 did not form in media allowing good growth, and, if they were formed in small numbers, their detection was usually obscured by the vegetative growth. When observed with reflected light, these sporangia appeared as small protuberances barely discernible from the growth mass. The best conditions for observing the sporangia were obtained with transmitted light in a compound microscope under magnifications between $\times 100$ and $\times 200$. Under these conditions, the sporangia formed on mineral agar with one of a number of carbon sources (see below) appeared as irregularly shaped bodies resembling sclerotia (Fig. 1). Detached sporangia had, however, a normal appearance in wet mounts (Fig. 2), where the release of motile spores was by the same mechanism as with other actinoplanetes.

Good formation of sporangia was observed in many of the plates of mineral medium with various carbon sources, including the following: L-rhamnose, D-galactose, D-fructose, lactose, gluconate, pimelate, L-aspartate, $\mathrm{D}$-aspartate, L-asparagine, L-histidine, L-tyrosine, L-phenylalanine, L-tryptophan, anthranilate, sarcosine, and acetamide. Copious formation of sporangia occurred in numerous instances in solid or liquid media with carbon sources which permitted very poor or no visible growth. Few sporangia were formed in the solid mineral medium not supplemented with a carbon source.

Release of motile spores from the sporangia is triggered by flooding the plates with water or buffer solutions. The globose spores measured 0.9 to $1.0 \mu \mathrm{m}$ in diameter and had a tuft of flagella inserted in a limited area of the cell (Fig. 3).

Analyses of the cell wall hydrolysates by paper chromatography demonstrated the presence of

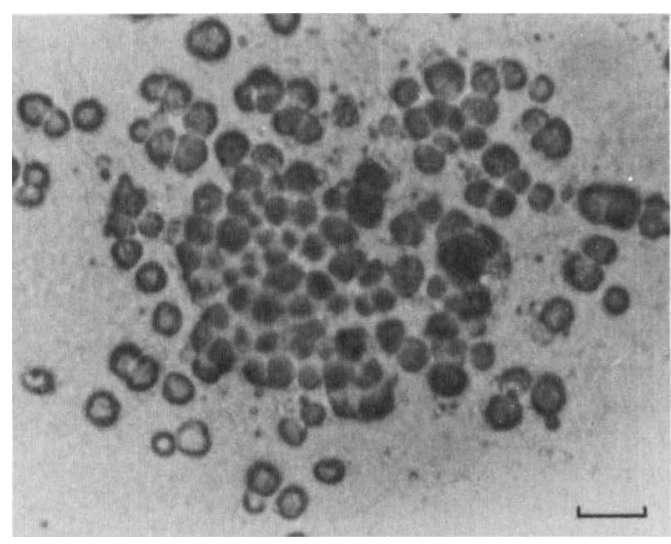

FIG. 1. Formation of sporangia by strain X-14695 on mineral agar medium with $0.1 \%$ L-aspartate as the sole carbon source. Bar represents $20 \mu \mathrm{m}$. 

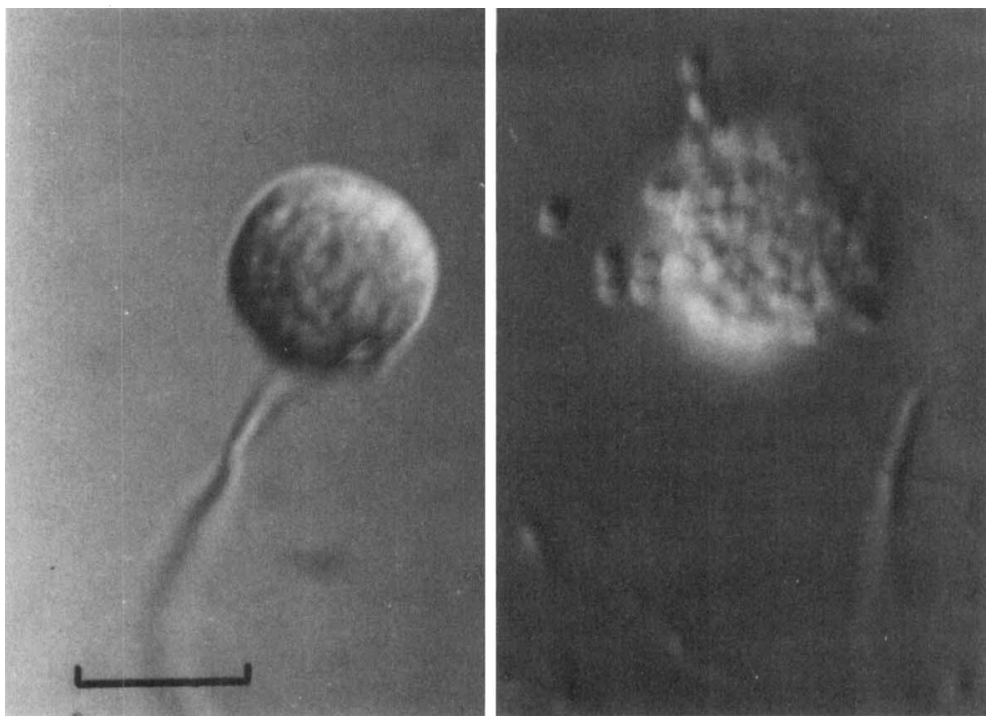

FIG. 2. Sporangia of strains $S-14695$. The sporangium at the right is releasing motile spores. Bar represents $10 \mu \mathrm{m}$.
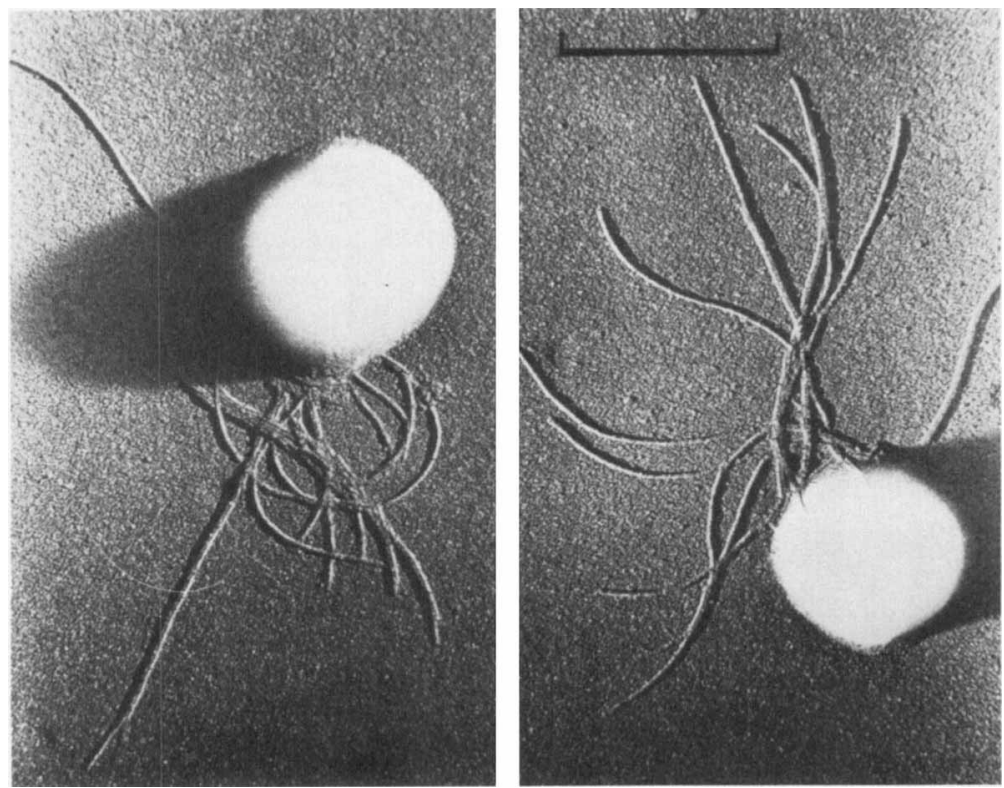

FIG. 3. Spores of strain $X \cdot 14695$. Bar represents $1 \mu \mathrm{m}$.

an isomer of diaminopimelic acid (DAP) other than the LL form. Presumably, this compound is the meso isomer, but no further discrimination from the DD isomer was attempted. In addition to meso-DAP, the hydrolysates also contained a lowly moving component which had the same staining properties with ninhydrin as did DAP. This component appeared in a smaller quantity than did DAP, and it is probably one of the isomers of 3-hydroxy-DAP. The $R_{f}$ of this com- pound was identical to that of the hydroxy-DAP of a hydrolysate of $A$. utahensis ATCC 14539 cell walls $(10,12)$. In addition, it was found that A. brasiliensis ATCC 25844 also contained meso- and/or DD-DAP and 3-hydroxy-DAP.

The morphological and chemical characteristics of strain X-14695 permit a clear diagnosis of this organism as a member of the genus Actinoplanes.

Growth characteristics and physiological 


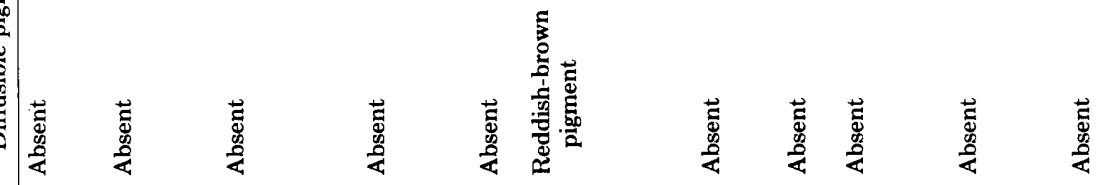

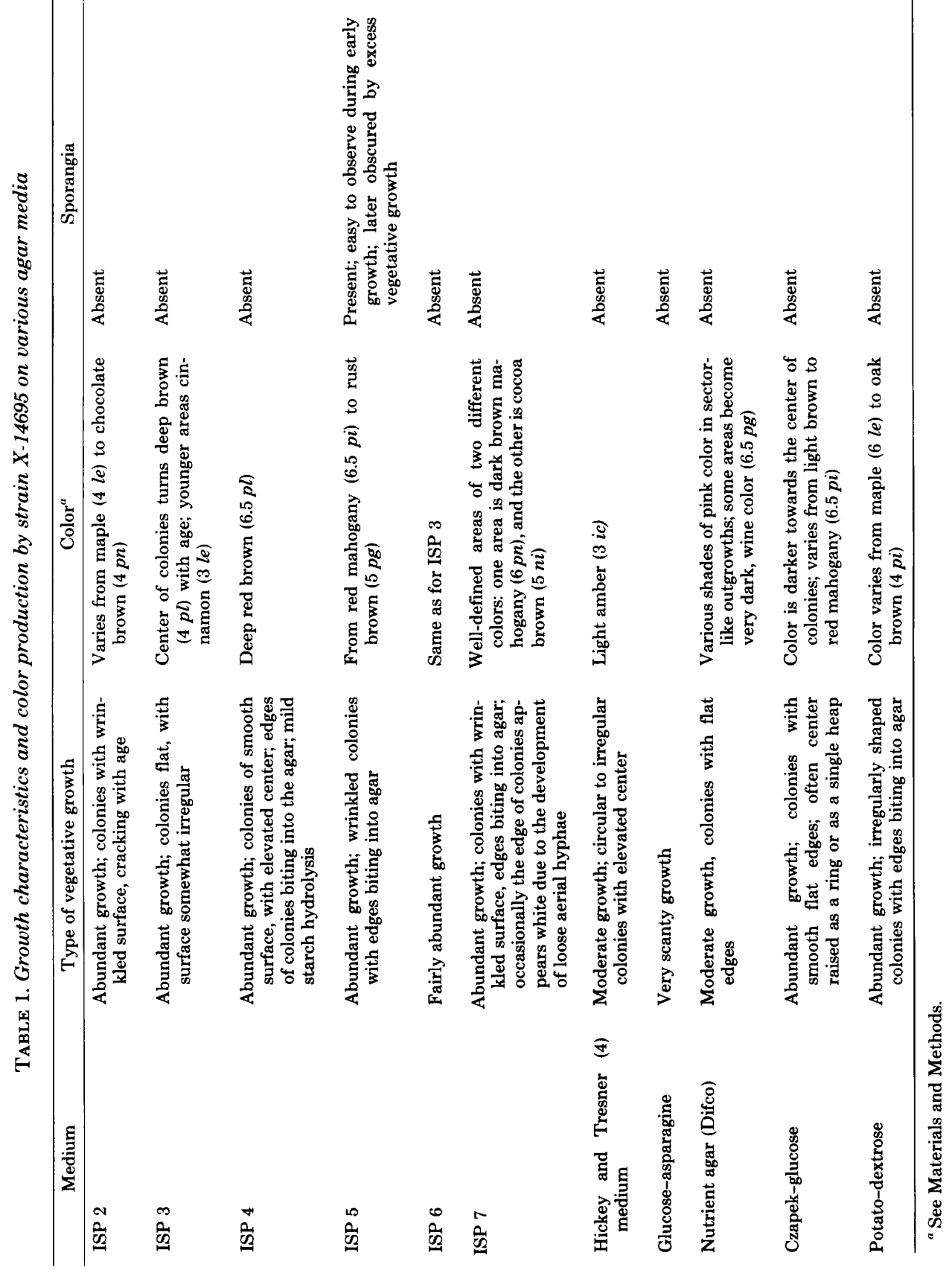


properties. Growth of strain X-14695 was very good at $32^{\circ} \mathrm{C}$ and somewhat less rapid at $28^{\circ} \mathrm{C}$. There was poor growth at $35^{\circ} \mathrm{C}$ and no growth at $37^{\circ} \mathrm{C}$. Growth was extremely poor at $10^{\circ} \mathrm{C}$.

Casein and gelatin were hydrolyzed. Growth was negative at $\mathrm{NaCl}$ concentrations of $5 \%$ or higher. Chemotactic experiments indicated a very moderate attraction of the spores toward chloride ions compared with the spores of $A$. brasiliensis ATCC 25844 (6).

Growth characteristics and color production in various media are given in Table 1.

No characteristic carotenoid spectrum was evident in a scan of a methanolic extract of cells. A major peak at $408 \mathrm{~nm}$ and a shoulder at 418 $\mathrm{nm}$ were the important characteristics of the absorption spectrum in the visible region.

Of 140 different carbon compounds, 29 supported good growth of strain X-14695. These compounds were $\mathrm{D}$-xylose, L-arabinose, $\mathrm{D}$-glucose, D-mannose, D-galactose, trehalose, maltose, cellobiose, lactose, starch, chitin, gluconate, acetate, succinate, fumarate, suberate, L-malate, pyruvate, glycerol, D-arabitol, mannitol, L-alanine, beta-alanine, L-threonine, L-aspartate, Lglutamate, L-asparagine, L-proline, and spermine. Growth was very slow on D-arabinose, Dfructose, salicin, lactobionate, propionate, sebacate, phenylacetate, and phenylalanine.

When compared with the nutritional spectra of $A$. brasiliensis, $A$. italicus, $A$. utahensis, $A$. philippinensis, $A$. missouriensis, and A. armeniacus, that of strain X-14695 appears to be quite restricted (B. Prosser and N. J. Palleroni, unpublished data). Strain X-14695 differs from strains of $A$. italicus, a red-pigmented species of the genus, in that the latter utilize many organic compounds as growth substrates. A. italicus grows on D-ribose, L-rhamnose, sucrose, betahydroxybutyrate, DL-lactate, citrate, alpha-ketoglutarate, $m$-inositol, quinate, $\mathrm{D}$-alanine, L-arginine, L-glutamine, gamma-aminobutyrate, and L-histidine. These substrates are not used by strain X-14695. Strain X-14695 grows on D-arabitol and spermine, which are not utilized by $A$. italicus. Moreover, the pigment produced by strain X-14695 remains, in most cases, associated with the colonies, and no deep cherry-red diffusible pigment on medium ISP 4 (2) is apparent.

The morphological and physiological properties of strain X-14695 are different from those of other members of the genus Actinoplanes. In addition, the organism has the capacity of producing the proline antimetabolite L-azetidine-2carboxylic acid. This antimetabolite was found to be produced by this strain and not by many hundreds of other strains of the same genus (Pruess and Scannell, personal communication). Therefore, strain X-14695 appears to be a member of a new species of the genus Actinoplanes, for which the name Actinoplanes ferrugineus is proposed (fer.ru.gi ne.us. L. adj. ferrugineus of the color of iron rust). Strain X-14695 is the type strain of $A$. ferrugineus. A culture of this strain has been deposited in the American Type Culture Collection under the number 29868.

\section{ACKNOWLEDGMENT}

The kind collaboration of J. Baker in sending many soil samples from various locations in Australia is gratefully acknowledged.

\section{REPRINT REQUESTS}

Address reprint requests to: Dr. Norberto J. Palleroni, Chemical Research Department, Hoffman-LaRoche, Inc., Nutley, NJ 07110.

\section{LITERATURE CITED}

1. Becker, B., M. P. Lechevalier, R. E. Gordon, and H. R. Lechevalier. 1964. Rapid differentiation between Nocardia and Streptomyces by paper chromatography of whole-cell hydrolysates. Appl. Microbiol. 12:421-423.

2. Beretta, G. 1973. Actinoplanes italicus, a new red-pigmented species. Int. J. Syst. Bacteriol. 23:37-42.

3. Fowden, L. 1976. Imino acids and N-hetero-cyclic amino acids from plants. Heterocycles 4:117-130.

4. Hickey, R. J., and H. D. Tresner. 1952. A cobalt-containing medium for sporulation of Streptomyces species. J. Bacteriol. 64:891-892.

5. Palleroni, N. J. 1976. Chamber for bacterial chemotaxis experiments. Appl. Environ. Microbiol. 32:729-730.

6. Palleroni, N. J. 1976. Chemotaxis in Actinoplanes. Arch. Microbiol. 110:13-18.

7. Prosser, B., and N. J. Palleroni. 1976. Streptomyces longwoodensis sp. nov. Int. J. Syst. Bacteriol. 26: 319-322.

8. Shirling, E. B., and D. Gottlieb. 1966. Methods for characterization of Streptomyces species. Int. J. Syst. Bacteriol. 16:313-340.

9. Skerman, V. B. D. 1967. A guide to the identification of the genera of bacteria. The Williams \& Wilkins Co., Baltimore.

10. Szaniszlo, P. J., and H. Gooder. 1967. Cell wall composition in relation to the taxonomy of some Actinoplanaceae. J. Bacteriol. 94:2037-2047.

11. Waksman, S. A. 1961. The actinomycetes, vol. 2. The Williams \& Wilkins Co., Baltimore.

12. Yamaguchi, T. 1965. Comparison of cell-wall composition of morphologically distinct actinomycetes. J. Bacteriol. 89:444-453. 